\title{
Basic science (December 2006)
}

1. Ahn J, Ambrosone CB, Kanetsky PA, Tian CQ, Lehman TA, Kropp S, Helmbold I, von Fournier D, Haase W, Sautter-Bihl ML, Wenz F, ChangClaude J. Polymorphisms in genes related to oxidative stress (CAT, MnSOD, MPO, and eNOS) and acute toxicities from radiation therapy following lumpectomy for breast cancer. Clin Cancer Res 2006; 12: 7063-7070.

2. Bates GJ, Fox SB, Han C, Leek RD, Garcia JF, Harris AL, Banham AH. Quantification of regulatory $T$ cells enables the identification of high-risk breast cancer patients and those at risk of late relapse. J Clin Oncol 2006; 24: 5373-5380.

3. Benusiglio PR, Pharoah PD, Smith PL, Lesueur F, Conroy D, Luben RN, Dew G, Jordan C, Dunning A, Easton DF, Ponder BAJ. HapMapbased study of the 17q21 ERBB2 amplicon in susceptibility to breast cancer. Br J Cancer 2006; 95: 1689-1695.

4. Bewick MA, Conlon MSC, Lafrenie RM. Polymorphisms in XRCC1, XRCC3, and CCND1 and survival after treatment for metastatic breast cancer. J Clin Oncol 2006; 24: 5645-5651.

5. Cheang MCU, Treaba DO, Speers $\mathrm{CH}$, Olivotto IA, Bajdik CD, Chia SK, Goldstein LC, Gelmon KA, Huntsman D, Gilks CB, Nielsen TO, Gown AM. Immunohistochemical detection using the new rabbit monoclonal antibody SP1 of estrogen receptor in breast cancer is superior to mouse monoclonal antibody 1D5 in predicting survival. $J$ Clin Oncol 2006; 24: 5637-5644.

6. Chen S, Oh SR, Phung S, Hur G, Ye IJ, Kwok SL, Shrode GE, Belury M, Adams LS, Williams D. Anti-aromatase activity of phytochemicals in white button mushrooms (Agaricus bisporus). Cancer Res 2006; 66: 12026-12034.

7. Chin K, DeVries S, Fridlyand J, Spellman PT, Roydasgupta R, Kuo WL, Lapuk A, Neve RM, Qian ZW, Ryder T, Chen FQ, Feiler H, Tokuyasu T, Kingsley C, Dairkee S, Meng ZH, Chew K, Pinkel D, Jain A, Ljung BM, Esserman L, Albertson DG, Waldman FM, Gray JW. Genomic and transcriptional aberrations linked to breast cancer pathophysiologies. Cancer Cell 2006; 10: 529-541.
8. Chlebowski RT, Blackburn GL, Thomson CA, Nixon DW, Shapiro A, Hoy MK, Goodman MT, Giuliano AE, Karanja N, McAndrew P, Hudis C, Butler J, Merkel D, Kristal A, Caan B, Michaelson R, Vinciguerra V, Del Prete S, Winkler M, Hall R, Simon M, Winters BL, Elashoff RM. Dietary fat reduction and breast cancer outcome: interim efficacy results from the Women's Intervention Nutrition Study. J Natl Cancer Inst 2006; 98: 1767-1776.

9. Cowell LN, D Graham J, Bouton AH, Clarke CL, O'Neill GM. Tamoxifen treatment promotes phosphorylation of the adhesion molecules, p130Cas/ BCAR1, FAK and Src, via an adhesion-dependent pathway. Oncogene 2006; 25: 7597-7607.

10. Desmedt C, El Ouriaghli F, Durbecq V, Soree A, Colozza MA, Azambuja E, Paesmans M, Larsimont $D$, Buyse $M$, Harris A, Piccart $M$, Martiat P, Sotiriou C. Impact of cyclins E, neutrophil elastase and proteinase 3 expression levels on clinical outcome in primary breast cancer patients. Int J Cancer 2006; 119: 2539-2545.

11. Esseghir S, Reis JS, Kennedy A, James $M$, O'Hare MJ, Jeffery R, Poulsom R, Isacke CM. Identification of transmembrane proteins as potential prognostic markers and therapeutic targets in breast cancer by a screen for signal sequence encoding transcripts. J Pathol 2006; 210: 420-430.

12. Fan MY, Yan PS, Hartman-Frey C, Chen L, Paik $\mathrm{H}$, Oyer SL, Salisbury JD, Cheng ASL, Li L, Abbosh PH, Huang THM, Nephew KP. Diverse gene expression and DNA methylation profiles correlate with differential adaptation of breast cancer cells to the antiestrogens tamoxifen and fulvestrant. Cancer Res 2006; 66: 11954-11966.

13. Gabos Z, Sinha R, Hanson J, Chauhan N, Hugh J, Mackey JR, Abdulkarim B. Prognostic significance of human epidermal growth factor receptor positivity for the development of brain metastasis after newly diagnosed breast cancer. J Clin Oncol 2006; 24: 5658-5663.

14. Garcia R, Franklin RA, McCubrey JA. EGF induces cell motility and multi-drug resistance gene expression in breast cancer cells. Cell Cycle 2006; 5: 2820-2826.

BCO/636/2007/JW 
15. Hartman J, Lindberg K, Morani A, Inzunza J, Strom A, Gustafsson JA. Estrogen receptor beta inhibits angiogenesis and growth of T47D breast cancer xenografts. Cancer Res 2006; 66: 11207-11213.

16. Huang Y, Li X, Jiang J, Frank SJ. Prolactin modulates phosphorylation, signaling and trafficking of epidermal growth factor receptor in human T47D breast cancer cells. Oncogene 2006; 25: 7565-7576.

17. Jansson AK, Gunnarsson C, Cohen M, Sivik T, Stal O. 17 beta-hydroxysteroid dehydrogenase 14 affects estradiol levels in breast cancer cells and is a prognostic marker in estrogen receptorpositive breast cancer. Cancer Res 2006; 66: 11471-11477.

18. Kilker RL, Planas-Silva MD. Cyclin D1 is necessary for tamoxifen-induced cell cycle progression in human breast cancer cells. Cancer Res 2006; 66: 11478-11484.

19. Kim HT, Kong G, DeNardo D, Li YX, Uray I, Pal S, Mohsin S, Hilsenbeck SG, Bissonnette R, Lamph WW, Johnson K, Brown PH. Identification of biomarkers modulated by the rexinoid LGD1069 (bexarotene) in human breast cells using oligonucleotide arrays. Cancer Res 2006; 66: 12009-12018.

20. Kouros-Mehr $H$, Slorach EM, Sternlicht MD, Werb Z. GATA-3 maintains the differentiation of the luminal cell fate in the mammary gland. Cell 2006; 127: 1041-1055.

21. Li HY, Weinstein IB. Protein kinase $\mathrm{C}$ beta enhances growth and expression of cyclin D1 in human breast cancer cells. Cancer Res 2006; 66: 11399-11408.

22. Lin EY, Li JF, Gnatovskiy L, Deng Y, Zhu L, Grzesik DA, Qian H, Xue XN, Pollard JW. Macrophages regulate the angiogenic switch in a mouse model of breast cancer. Cancer Res 2006; 66: 11238-11246.

23. Liu Y, Smith PW, Jones DR. Breast cancer metastasis suppressor 1 functions as a corepressor by enhancing histone deacetylase 1-mediated deacetylation of RelA/p65 and promoting apoptosis. Mol Cell Biol 2006; 26: 8683-8696.

24. Ma ZF, Gibson SL, Byrne MA, Zhang JR, White MF, Shaw LM. Suppression of insulin receptor substrate 1 (IRS-1) promotes mammary tumor metastasis. Mol Cell Biol 2006; 26: 9338-9351.

25. Nakagawa T, Huang SK, Martinez SR, Tran AN, Elashoff D, Ye X, Turner RR, Giuliano AE, Hoon DSB. Proteomic profiling of primary breast cancer predicts axillary lymph node metastasis. Cancer Res 2006; 66: 11825-11830.

26. Neve RM, Chin K, Fridlyand J, Yeh J, Baehner FL, Fevr T, Clark L, Bayani N, Coppe JP, Tong F,
Speed T, Spellman PT, DeVries S, Lapuk A, Wang NJ, Kuo WL, Stilwell JL, Pinkel D, Albertson DG, Waldman FM, McCormick F, Dickson RB, Johnson MD, Lippman M, Ethier S, Gazdar A, Gray JW. A collection of breast cancer cell lines for the study of functionally distinct cancer subtypes. Cancer Cell 2006; 10: 515-527.

27. Poole AJ, Li Y, Kim Y, Lin SCJ, Lee WH, Lee E. Prevention of Brca1-mediated mammary tumorigenesis in mice by a progesterone antagonist. Science 2006; 314: 1467-1470.

28. Porter PL, Barlow WE, Yeh IT, Lin MG, Yuan XPP, Donato E, Sledge GW, Shapiro CL, Ingle JN, Haskell CM, Albain KS, Roberts JM, Livingston RB, Hayes DF. p27(Kip1) and cyclin E expression and breast cancer survival after treatment with adjuvant chemotherapy. $J$ Natl Cancer Inst 2006; 98: 1723-1731.

29. Rosenberg LU, Einarsdottir K, Friman EI, Wedren S, Dickman PW, Hall P, Magnusson C. Risk factors for hormone receptor-defined breast cancer in postmenopausal women. Cancer Epidemiol Biomark Prevent 2006; 15: 2482-2488.

30. Salatino M, Beguelin W, Peters MG, Carnevale R, Proietti CJ, D Galigniana M, Vedoy CG, Schillaci R, Charreau EH, Sogayar MC, Elizalde PV. Progestininduced caveolin-1 expression mediates breast cancer cell proliferation. Oncogene 2006; 25: 7723-7739.

31. Schneider J, Ruschhaupt M, Buness A, Asslaber M, Regitnig P, Zatloukal K, Schippinger W, Ploner F, Poustka A, Sultmann H. Identification and meta-analysis of a small gene expression signature for the diagnosis of estrogen receptor status in invasive ductal breast cancer. Int J Cancer 2006; 119: 2974-2979.

32. Setiawan VW, Cheng IN, Stram DO, Giorgi E, Pike MC, Van Den Berg D, Pooler L, Burtt NP, Le Marchand L, Altshuler D, Hirschhorn J, Henderson BE, Haiman CA. A systematic assessment of common genetic variation in CYP11A and risk of breast cancer. Cancer Res 2006; 66: 12019-12025.

33. Thompson D, Seal S, Schutte M, McGuffog L, Barfoot R, Renwick A, Eeles R, Sodha N, Houlston R, Shanley S, Klijn J, Wasielewski M, Chang-Claude J, Futreal PA, Weber BL, Nathanson KL, Stratton M, Meijers-Heijboer H, Rahman N, Easton DF. A multicenter study of cancer incidence in CHEK2 1100delC mutation carriers. Cancer Epidemiol Biomark Prevent 2006; 15: 2542-2545.

34. Torres-Arzayus MI, Yuan J, DellaGatta JL, Lane H, Kung AL, Brown M. Targeting the AIB1 oncogene through mammalian target of rapamycin inhibition 
in the mammary gland. Cancer Res 2006; 66: 11381-11388.

35. Wang LH, Yang $X Y$, Zhang $X H$, An P, Kim HJ, Huang JQ, Clarke R, Osborne CK, Inman JK, Appella E, Farrar WL. Disruption of estrogen receptor DNA-binding domain and related intramolecular communication restores tamoxifen sensitivity in resistant breast cancer. Cancer Cell 2006; 10: 487-499.

36. Yang CW, Trent S, lonescu-Tiba V, Lan L, Shioda T, Sgroi D, Schmidt EV. Identification of cyclin D1-and estrogen-regulated genes contributing to breast carcinogenesis and progression. Cancer Res 2006; 66: 11649-11658.

37. Yang ZQ, Streicher KL, Ray ME, Abrams J, Ethier SP. Multiple interacting oncogenes on the 8p11-p12 amplicon in human breast cancer. Cancer Res 2006; 66: 11632-11643.

38. Yen L, Cao ZW, Wu XL, Ingalla ERQ, Baron C, Young LJT, Gregg JP, Cardiff RD, Borowsky AD,
Sweeney C, Carraway KL. Loss of Nrdp1 enhances ErbB2/ErbB3-dependent breast tumor cell growth. Cancer Res 2006; 66: 11279-11286.

39. Yook JI, Li XY, Ota I, Hu C, Kim HS, Kim NH, Cha SY, Ryu JK, Choi YJ, Kim J, Fearon ER, Weiss SJ. A Wnt-Axin2-GSK3 beta cascade regulates Snail1 activity in breast cancer cells. Nat Cell Biol 2006; 8: 1398-1406.

40. Zinser GM, Leonis MA, Toney K, Pathrose P, Thobe M, Kader SA, Peace BE, Beauman SR, Collins MH, Waltz SE. Mammary-specific Ron receptor overexpression induces highly metastatic mammary tumors associated with beta-catenin activation. Cancer Res 2006; 66: 11967-11974.

Prepared by R. Sutherland, J. Scorer Cancer Research Program Garvan Institute of Medical Research Darlinghurst, NSW, Australia 